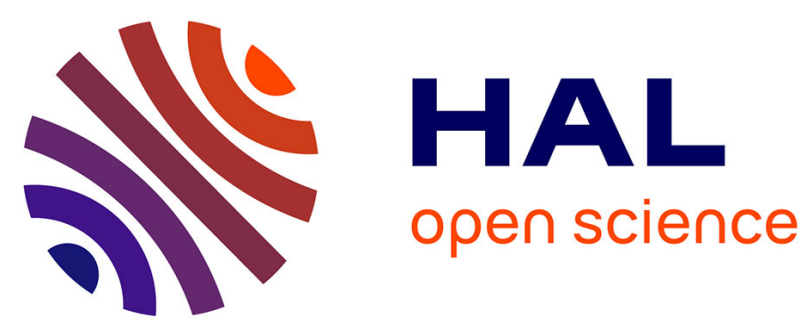

\title{
Robotic micromanipulation for microassembly: modelling by sequencial function chart and achievement by multiple scale visual servoings.
}

Brahim Tamadazte, Nadine Lefort-Piat, Sounkalo Dembélé, Guillaume Fortier

\section{- To cite this version:}

Brahim Tamadazte, Nadine Lefort-Piat, Sounkalo Dembélé, Guillaume Fortier. Robotic micromanipulation for microassembly: modelling by sequencial function chart and achievement by multiple scale visual servoings.. Journal of Micro-Nano Mechatronics, 2009, pp.1-12. 10.1007/s12213-009-0019-0 . hal-00430977

\section{HAL Id: hal-00430977 https://hal.science/hal-00430977}

Submitted on 10 Nov 2009

HAL is a multi-disciplinary open access archive for the deposit and dissemination of scientific research documents, whether they are published or not. The documents may come from teaching and research institutions in France or abroad, or from public or private research centers.
L'archive ouverte pluridisciplinaire HAL, est destinée au dépôt et à la diffusion de documents scientifiques de niveau recherche, publiés ou non, émanant des établissements d'enseignement et de recherche français ou étrangers, des laboratoires publics ou privés. 


\section{Introduction}

\section{Robotic micromanipulation for microassembly: modelling by sequencial function chart and achievement by multiple scale visual servoings}

\author{
Brahim Tamadazte . \\ Nadine Le Fort-Piat . \\ Sounkalo Dembélé . \\ Guillaume Fortier
}

Received: August 28, 2009 / Accepted: September 27, 2009

\begin{abstract}
The paper investigates robotic assembly by focusing on the manipulation of microparts. This task is formalized through the notion of basic tasks which are organized in a logical sequence represented by a function chart and interpreted as the model of the behavior of the experimental setup. The latter includes a robotic system, a gripping system, an imaging system, and a clean environment. The imaging system is a photon videomicroscope able to work at multiple scales. It is modelled by a linear projective model where the relation between the scale factor and the magnification or zoom is explicitly established. So, the usual visual control law is modified in order to take into account this relation. The manipulation of some silicon microparts $(400 \mu \mathrm{m} \times 400 \mu \mathrm{m} \times 100 \mu \mathrm{m})$ by means of a distributed robotic system ( $x y \theta$ system, $\varphi \mathrm{z}$ system), a two-finger gripping system and a controllable zoom and focus videomicroscope shows the relevance of the concepts. The $30 \%$ of failure rate comes mainly from the physical phenomena (electrostatic and capillary forces) instead of the accuracy of control or the occultations of microparts.
\end{abstract}

Keywords micromanipulation - manipulation sequence · multiple scale calibration - multiple scale visual servoing · visual tracking

FEMTO-ST Institute, UMR CNRS 6174 - UFC / ENSMM / UTBM. Automatic Control and Micro-Mechatronic Systems Department 24 rue Alain Savary, 25000 Besançon, France

Tel.: +33 (0)3 814027 97, Fax: +33 (0)381402809

E-mail: correspending author, brahim.tamadazte@ens $2 \mathrm{~m} . \mathrm{fr}$
Microassembly stands for assembly of micrometric (i.e. submillimeter) parts in order to obtain compound products, more particularly in three dimensions. The size of those objects may not be micrometric, the important point is the fact that they include at least one micrometric part. They can be sensors, actuators as well as structures and are developed for a large amount of applications in biomedical field, aerospace engineering, automotive households, IT Peripherals, etc. The microassembly process can be classified into self-assembly and robotic assembly. In the former case, physical phenomena (mechanical, electrostatic,...) and chemical bonds enable the joining of the various components and require the functionalization of the different surfaces that intervene in the process. In the second case, a robotic system usually in combination with a gripping system and an imaging system are used to reach the objective. The microassembly process can be break down into basic tasks that are sequentially performed: visual detection of the microcomponent, positioning of the microcomponent, positioning of the endeffector, gripping of the microcomponent, transfer of the microcomponent, release of the microcomponent, etc. This paper leads with the second approach i.e. robotic micromanipulation and microassembly. The size of microparts involved in the process of microassembly is very important. The more the size decreases the more difficult is the manipulation since the requirement of tolerance, resolution and accuracy increase and the effect of adhesion becomes predominant over that of usual gravity. The size of $10 \mu \mathrm{m}$ is a threshold. Below this value, the gravity force becomes the least important force of all ([14], [17]). The microassembly or micromanipulation process can be performed in different modes: automatic mode (using vision or force feedback), semi-automatic mode (only a part of the process is automated), tele-operated mode (using a joystick) and manual mode.

\subsection{Overview}

Among the works mentioned in the literature dealing with the problem of automation of such tasks, we can cite the fully automated robotic insertion of a $228.6 \mu \mathrm{m}$ diameter peg into a hole of $254 \mu \mathrm{m}$ diameter which was achieved with an accuracy of $2.2 \mu \mathrm{m}$ by B. J. Nelson and his colleagues ([44], [50], [27], [33]). More precisely, in this work a photon videomicroscope with four magnifications $(2 \times, 10 \times, 20 \times$, $50 \times)$ is used enabling authors to implement a four-scale visual servoing of type of LQ (Linear quadratic) control law. The process switches successively from $2 \times$ magnification at the beginning to $50 \times$ at the end. In parallel, insertion of a $100 \mu \mathrm{m}$ diameter peg into a hole was performed by Chen et $a l$. ([6]) and that of a $500 \mu \mathrm{m} \times 20 \mu \mathrm{m}$ into hole of 
$550 \mu \mathrm{m} \times 500 \mu \mathrm{m}$ was done by Enikov et al. ([12]). In the same case, insertion of a $450 \mu \mathrm{m} \times 400 \mu \mathrm{m} \times 300 \mu \mathrm{m}$ peg into a hole of $650 \mu \mathrm{m} \times 500 \mu \mathrm{m} \times 300 \mu \mathrm{m}$ was performed by Tao et al. ([41]) with an active zooming of the microscope during the manipulation. Of course this is also done with a multiple scale visual servoing. Lee et al. ([24]) has worked about images-based micropositioning for 3D assembly of MEMS using a multiple scale approach. J. Wason et al. ([46]) have presented an automatic insertion of a $300 \mu \mathrm{m}^{2}$ micropart into a hole which was performed by the cooperation of three independent probes acting as fingers. In another case, semi-automated experiments of assembly metal microparts with a silicon microparts were reported by Yang et $a l$. ([18], [48]). In these experiments, the core of the task was the insertion of $500 \mu \mathrm{m} \times 100 \mu \mathrm{m}$ pegs into holes of $520 \mu \mathrm{m} \times 110 \mu \mathrm{m}$. The means included a distributed robotic and a four-view imaging systems.

After the peg-in-hole task, the assembly of some usual compound products was achieved. A manual experiment of assembly of a $1 \mathrm{~mm}^{3}$ house was conducted by Tsuchiya et al. ([42]). Four faces and the roof in two microparts were mounted and soldered in a SEM (Scanning Electron Microscope) chamber. We can also refer to the manual assembly of a $900 \mu \mathrm{m}$ diameter ball bearing with six components (1 housing, 6 balls of $100 \mu \mathrm{m}$ diameter, 1 shaft, 1 cover) which were achieved by Ashida et al. ([3]) and that of a gear train by Ogawa ([28]) in the case of microfactory paradigm whose main results were summarized by Okazaki in 2004 ([29]). This paradigm appeared less relevant in microassembly in opposition to manufacturing since the imaging system could not be miniaturizing. Another manual microassembly of 3D micro coils were reported by Dechev et al. ([9], [8]). In these works, passive silicon grippers and microparts were designed to exhibit compliance which enabled accurate assembly: simultaneous insertion of two tips $4 \mu \mathrm{m}$ wide into holes which were slightly smaller. The overall size of the micropart varied from $60 \mu \mathrm{m} \times 70 \mu \mathrm{m}$ to $300 \mu \mathrm{m} \times 400 \mu \mathrm{m}$. A distributed robotic system with a photon videomicroscope $(20 \times$ magnification) were used to carry out the experiments. The importance of suitable illumination for silicon microparts is pointed out. The authors recommend the use of light with a wavelength higher than $660 \mathrm{~nm}$. Udeshi et al. ([43]) and Saini et al. ([35]) reported assembly of micro columns using compliant systems as in the works of Dechev et al. ([8]).

Part of a genuine watch gear system including six components (plate, minute gear and pinion, hour gear and pinion, third gear and pinion) was automatically assembled by S. Koelemeijer et al. ([7]). More recently, assembly of a gear system including nine components ( 2 shafts, 5 gears, 1 wheel, 1 toothed base) was reported by Sun et al. ([38]) and Xie et al. ([47]). In this assembly, the diameter of the overall system was about $2 \mathrm{~mm}$. To perform the microassembly, a visual servoing with a modified Smith predictor enabling the modelling of the delay in the assembly process was implemented. Probst et al. ([32]) reported the mounting and fixing by UV activated glue of the structure of a microrobot with their novel microassembly system.

In the optical microsystems (also called MOEMS) assembly field, the assembly of compound products for photonic purpose was achieved. The partially automated assembly of a laser diode pump was achieved by Lee et $a l$. ([23]), Kim et al. ([22], [21]). The core task was the insertion of an optic fiber into the hole of a $400 \mu \mathrm{m} \times 300 \mu \mathrm{m} \times 90$ $\mu \mathrm{m}$ support. An automatic accomplishment was reported by Enikov et al. ([11]) by introducing the incompressible time of image formation and the retrieval of information as a delay in the control loop. Insertion of optic fibers into Vgroove was performed by Popa et al. ([30], [31]). Also in the MOEMS assembly field, microspheres of diameter about $1 \mu \mathrm{m}$ and plates of $25 \mu \mathrm{m} \times 25 \mu \mathrm{m} \times 0.5 \mu \mathrm{m}$ were manually assembled into 3D photonic crystals by Aoki et $a l$. published in the prestigious scientific revue Nature ([2]). The process was achieved by a microrobotic system in conjunction with a stereo SEM imaging system. This work led De La Rue ([34]) to conclude that microassembly is a promising approach to building the next generation of photonic devices.

Interesting works related to the manipulation and assembly of microbiomedical components are proposed in the literature. We can cite the assembly of microelectrode arrays for use in the central nervous system proposed by Ghovanloo et al. ([19]). The work consisted in stacking into a glass, planar micromachined probes with spacers (glass sheets or polyimide) in between. The spacer thickness varied from $250 \mu \mathrm{m}$ to $500 \mu \mathrm{m}$. Recently Sieber et al. ([36]) reported the microassembly (handling, positioning and bonding) of a force sensor $(1.5 \mathrm{~mm} \times 1.5 \mathrm{~mm} \times 0.65 \mathrm{~mm})$ for use in biomedical devices.

Beside the above experiments of microassembly, some authors have investigated specific visual tracking and servoing for microassembly compelling to deal with the photon videomicroscope modelling and calibration ([15], [50], [39], [16], [40]). As assembly on the micro scale is the continuation of assembly on the macro scale, developments achieved in visual tracking ([26]) or servoing ([5], [4]) are of high interest in microassembly.

Some authors have investigated the problem of organizing optimally the control architecture of the microassembly system by proposing a multiple level paradigm ([13], [25]).

Therefore, this state of the art of robotic microassembly leads to the conclusions exposed below.

1. Involved robotics systems are distributed. More precisely, the degrees of freedom (dof) are distributed to multiple autonomous systems which act together, 
2. Visual servo is an incontrovertible control scheme as image is the main source of information,

3. Control of the handling force is very relevant,

4. Multiple scale imaging is required in order to solve the classic field-of-view/resolution dilemma (a wide fieldof-view is incompatible with a high resolution) of the imaging system,

5. Control of part handling is indispensable, directly or via the environment.

6. Precision obtained during the micromanipulation and microassembly tasks is often superior to $2 \mu \mathrm{m}$.

\subsection{Contributions and organization}

From the overview presented above, it is appears that most of microassembly workcells is equipped a with distributed vision system; two or more optical microscopes (one top view camera and one or more lateral view cameras) ( [6], [33], This configuration is adapted to access to the $3 \mathrm{D}$ information of the scene during the manipulation or assembly tasks. In this paper, the distributed imaging system is replaced by a single optical videomicroscope for increasing considerably the workspace around the microassembly area. As regards, the computation of the $3 \mathrm{D}$ information is obtained using a depth-from-focus and pose-from-focus methods. Thanks to motorized zoom and focus of the photonic microscope, dynamic and multiple scale visual servoing is proposed. So, the new control laws presented in this paper integrate the zoom control in the image jacobian.

This paper investigates robotic assembly and it focuses more precisely on the manipulation of microparts. The concept is formalized through the notion of basic tasks which should be organized in a logical sequence. The latter is represented by a function chart (a Petri net is also possible) and interpreted as the model of the behavior of the experimental setup, which should include a robotic, a gripping and an imaging system and a clean environment. The imaging system consists on a photon videomicroscope which works at a multiple scale. It is modelled by a linear projective model where the relation between the scale factor and the magnification is explicitly established. Then, usual visual control laws are modified in order to take into account that relation. The manipulation of some silicon microparts $(400 \mu \mathrm{m} \times 400 \mu$ by means of a distributed robotic system ( $\mathrm{xy} \theta$ system, $\varphi \mathrm{z}$ system), a two-finger gripping system and a controllable zoom and focus videomicroscope shows the relevance of the concepts. Section II exposes the modelling of the manipulation task as a sequence of basic tasks which is represented by a function chart. Section III develops the multiple scale visual control by considering two laws: exponential and polynomial decrease of error. A multiple scale modelling of the photon videomicroscope is proposed in section IV. The corresponding calibration approach combining robotic and vi- sion is also exposed indeed usual practical calibration methods are not usable because of some properties of the microscope in comparison with a conventional lens. Section V exposes the vision algorithms used in the experiments. They include the tracking of micropart and gripper in the images of the scene, the autofocus achievement and the recovery of depth. The experimental setup is presented in section VI and the results of experimentations are exposed and analyzed in section VII. They show the relevance of the proposed concepts. Despite the use of a controlled environment room the electrostatic and capillary forces are high enough to cause the failure of the micromanipulation task.

\section{Modelling of robotic micromanipulation}

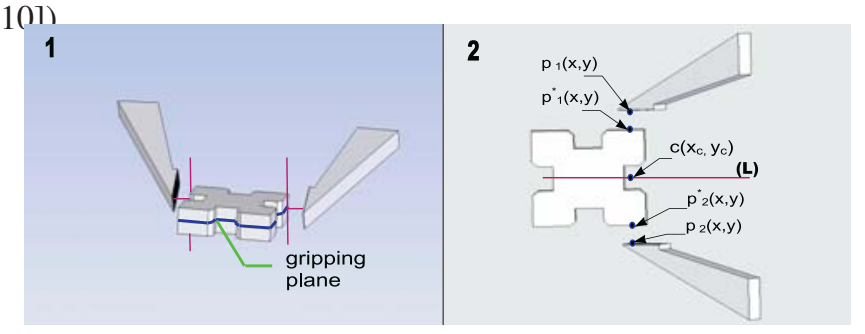

Fig. 1 View of the gripping plane and view of both of the gripping points.

Robotic manipulation corresponds to the handling and positioning of microparts by means of a robotic system in conjunction with a gripping system and an imaging system. As a consequence, the micromanipulation task can be broken down into basic tasks which must be performed in an ordered sequence. Some basic tasks are relative to the imaging system.

1. Task 1: Autofocusing. This task enables to keep focused the micropart of the images of the scene during the process.

2. Task 2: Detection of the micropart. The gripping of the micropart assumes its recognition and localization in the scene. If this scene includes several microparts, then it

$\times 100$ s $/$ neccessary to define the criteria for the selection of the target micropart.

3. Task 3: Increasing the imaging scale. A high scale of imaging and then of control enables a high accuracy of positioning.

4. Task 4: Decreasing the imaging scale. A low scale of imaging enables a global view of the work scene.

5. Task 5: Aligning of the micropart with the gripper fingers using the angular motion $\theta$ of the positioning platform. This task angles the micropart in the direction of the gripper fingers enabling its future grab. 
6. Task 6: Positioning and centering of the micropart with respect to the gripper. The central micropart is brought over the mid-point of the two finger-tips with an accuracy which is as high as possible (Fig. 1.1).

7. Task 7: Opening of the gripper. The gripper fingers are opened in order to encircle the micropart.

8. Task 8: Descent of the gripper. It is assumed that at the beginning the gripper is over the micropart, the gripping requires the aligning of the gripper fingers with the gripping plane of the micropart.

9. Task 9: Closing of the gripper. Every finger-tip (respectively $p_{1}$ and $p_{2}$ for the higher and lower tip) is displaced into a contact point (respectively $p_{1}^{*}$ and $p_{2}^{*}$ ) located in the gripping plane of the part (Fig. 1.2).

10. Task 10: Gripping of the part. An appropriate tightening force is required which enables the gripping without destruction of both the part and the gripper. That possible consequence justifies the implantation of force sensors in the gripper.

11. Task 11: Unsticking of the micropart of the support. A force is applied to the micropart that overcomes the adhesion forces between the part and the support.

12. Task 12: Ascent of the gripper with the micropart.

13. Task 13: Transfer of the micropart to the target location. The initial and final locations may be far enough which justifies a macro-micro motion paradigm.

14. Task 14: Release of the micropart. This task is decisive in the process. Because of the high level of capillary forces, the micropart trends to stick on the fingers after their opening. The performing of direct (gripper or part control) or indirect (environment control) strategies to make the release reliable is consequently strongly recommended.

Several logical combinations of the above basic tasks can lead to the desired result according to the systems available for the performance of the micromanipulation task. Each combination which is a sequence of the tasks can be specified by a function chart. Let the purpose of the primary (manipulation) task be a cyclical pick up of microparts from the initial locations and their place in target locations. The function chart of Fig. 2 is the representation of a sequence enabling the achievement of the pick and place task. In this case an additional basic task is associated corresponding to the motion of return of the gripper to its original location. Some tasks require a high accuracy, so it is recommended to check up on their success. The function chart representing the sequence can include that possibility as exposed in Fig. 2.

A sequence of tasks can be considered as a model for the behavior of the systems dedicated to perform the manipulation task. Consequently it can be used as a mean to perform the control of those systems. The manipulation appears as a sequence of servo controls including visual controls. The latter is of the multiple scale type as the imaging is of that type.

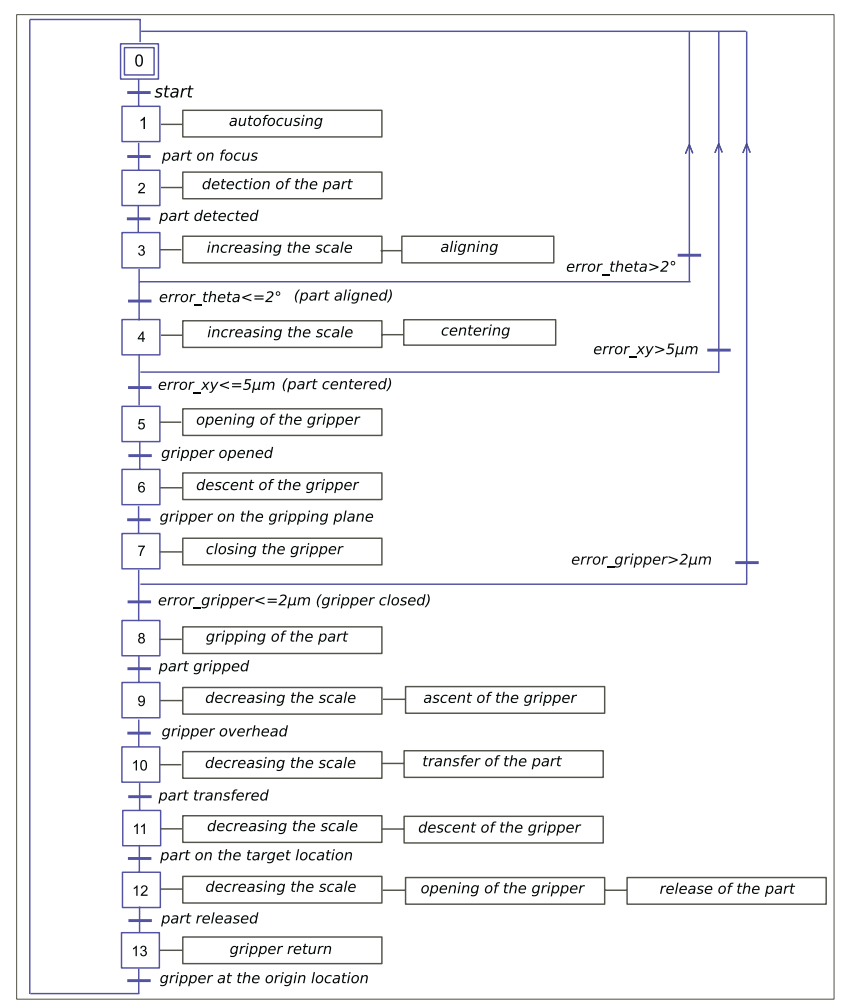

Fig. 2 Sequencial function chart of the picking and placing of a component.

\section{Multiple scale visual control}

Visual control in the image, more particularly 2D visual servoing, is accurate and robust to the errors of modelling of the imaging and the robotic systems. Thus, it is suitable for the achievement of basic manipulation tasks. Among the various numbers of control laws: exponential and polynomial decrease of error exhibit high efficiency.

\subsection{Exponential Decrease of error with adaptive gain}

The multiple scale paradigm is introduced in the control by explicitly modelling the scale factor $k$ as a function of the magnification or zoom factor $\zeta$. As a consequence, the focal length $(f)$, the desired value of the visual feature points $\left(\mathbf{s}^{*}\right)$ and the current value of those points (s) are functions of the zoom factor $\zeta: f(\zeta), \mathbf{s}^{*}(\zeta), \mathbf{s}(\zeta)$ (see 3 ). Therefore, the velocity $\dot{\mathbf{s}}$ of those visual features are linked to the relative velocity of the camera/scene $v$ by the following equation:

$\mathbf{s}^{*}=\mathbf{L}_{\mathbf{s}}\left(\mathbf{s}, Z_{f}, \zeta\right) \mathbf{v}$ 


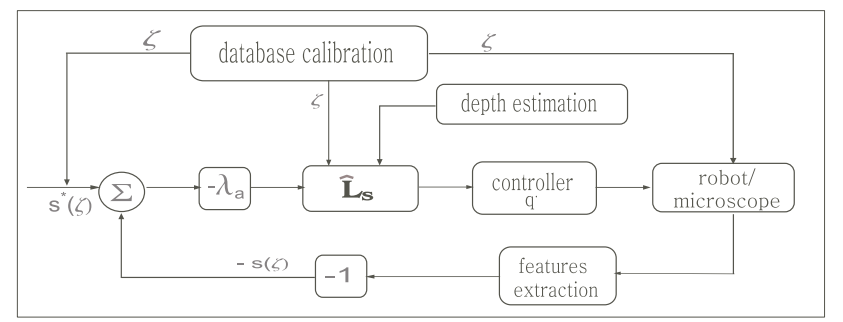

Fig. 3 Function chart of multiscale visual servoing with exponential decrease of error.

where $\mathbf{v}=\left[v_{x}, v_{y}, v_{z}, w_{x}, w_{y}, w_{z}\right]$ is the kinematic screw. $Z_{f}$ represents depth information of the considered object expressed in the camera frame.

The $\mathbf{L}_{\mathbf{s}}$ is the interaction matrix. For the normalized coordinates $x$ and $y$ respectively it can be written:

$\mathbf{L}_{\mathbf{s}}\left(\mathrm{x}, \mathrm{Z}_{f}, \zeta\right)=\left[\frac{-1}{\mathrm{Z}_{f}} 0 \frac{\mathrm{x}(\zeta)}{\mathrm{Z}_{f}} \mathrm{x}(\zeta) \mathrm{y}(\zeta)-1-\mathrm{x}(\zeta)^{2} \mathrm{y}(\zeta)\right]$

$\mathbf{L}_{\mathbf{s}}\left(\mathrm{y}, \mathrm{Z}_{f}, \zeta\right)=\left[0 \frac{-1}{\mathrm{Z}_{f}} \frac{\mathrm{y}(\zeta)}{\mathrm{Z}_{f}} 1+\mathrm{y}(\zeta)^{2}-\mathrm{x}(\zeta) \mathrm{y}(\zeta)-\mathrm{x}(\zeta)\right]$

Let $\mathbf{e}$ be the function task to regulate to zero:

$\mathbf{e}=\left(\mathbf{s}(\zeta)-\mathbf{s}^{*}(\zeta)\right)$

The exponential decrease of the function task e:

$\dot{\mathbf{e}}=-\lambda_{a} \mathbf{e}$

Leads to the following control law:

$\mathbf{v}=-\lambda_{a} \widehat{\mathbf{L}}_{\mathbf{s}} \mathbf{e}$

with $\lambda_{a}$ is a positive gain. To improve the convergence rate, we have implemented an adaptive gain (the gain increases when the error decreases):

$\lambda_{\text {adp }}=\left(\lambda_{\text {max }}-\lambda_{\text {min }}\right) \exp ^{-\kappa\|\mathbf{e}\|}+\lambda_{\text {min }}$

where $\lambda_{\max }$ and $\lambda_{\min }$ are respectively the maximum and minimum values of $\lambda_{\mathrm{adp}}, \kappa$ is a constant.

\subsection{Polynomial decrease of error}

The control law consists in regulating toward zero the distance between the desired value $\mathbf{s}_{f}^{*}$ and the current value $\mathbf{s}$ of a feature point. Let the distance corresponding to the task function be $e_{1}$ :

$\mathbf{e}_{1}=\delta\left(\mathbf{s}_{f}^{*}-\mathbf{s}\right)$
The polynomial decrease of that function leads to the following control:

$U_{d 1}(t)=\frac{\mathbf{e}_{1}(t)}{N}$

with $N$ as a constant integer.

\section{Multiscale modelling of the photon videomicroscope}

\subsection{Basic model}

The standard lens based image source is modelled by the non linear projective model. The latter includes: intrinsic parameters inherent to the imaging system e.g. the focal length $\boldsymbol{f}$, the scale factors $k_{x}, k_{y}$ in $\mathbf{x}$ and $\mathbf{y}$ directions, the principal point coordinates $\left(x_{o}, y_{o}\right)$ (front focal point) and extrinsic parameters corresponding to the position and orientation of the focusing element (usually called camera) frame with respect to the scene frame: the translation components $T_{x}, T_{y}$, and $T_{z}$, and for example Euler angles $\alpha, \beta$ and $\gamma$ and distortion parameters: the radial $\left(a_{1}, a_{2}, \ldots\right)$ and tangential $\left(b_{1}, b_{2}, \ldots\right)$ distortion coefficients. Because the improvement of technology enables the manufacturing of isotropic image sensors in which the scale factors along $x$ and $y$ are identical:

$k_{x}=k_{y}=k$

The microscope based image source is an optical imaging and thus can be modelled by a non linear projective model. However, the quality of a laboratory microscope is usually better than that of a standard lens: experimental values of distortion parameters are very weak, they range from $10^{-8}$ to $10^{-10}$ ([49], [1]). As a consequence the distortion can be neglected and so the videomicroscope can be modelled by the linear projective model. So, the model consists in a perspective projection of a scene point $P$ onto a pixel $p$ in the retinal plane through the optical center. Let $P$ and $p$ be respectively represented by the homogeneous vector $(X, Y, Z, 1)^{T}$ and $(x, y, w)^{T}$, the model can be written:

$p=Q P$

The matrix $Q$, dimension $3 \times 4$, is the homogeneous projection matrix of the image source. It is written:

$Q=K\left[\begin{array}{llll}1 & 0 & 0 & 0 \\ 0 & 1 & 0 & 0 \\ 0 & 0 & 1 & 0\end{array}\right] D$

The matrix $K$ gathers the intrinsic parameters as followed: 
$K=\left(\begin{array}{ccc}f k & 0 & x_{o} \\ 0 & f k & y_{o} \\ 0 & 0 & 1\end{array}\right)$

In a microscope where a tube is associated with the lens, the parameter $f$ in above equations becomes the sum of the focal length and the length of that tube.

The matrix $D$ combines the rotation matrix $[\mathbf{R}]$ and the translation vector $[\mathbf{T}]$ as follows:

$D=\left(\begin{array}{cc}R_{3 \times 3} & T_{1 \times 3} \\ 0 & 1\end{array}\right)$

If the Euler angles $\alpha, \beta$, and $\gamma$ are considered, that rotation matrix will be formulated in (15), see Fig. 4 .

$\mathbf{R}=\left(\begin{array}{ccc}\cos \alpha \cos \beta & \cos \alpha \sin \beta \sin \gamma-\sin \alpha \cos \gamma & \cos \alpha \sin \beta \sin \gamma+\sin \alpha \sin \gamma \\ \sin \alpha \cos \beta & \sin \alpha \sin \beta \sin \gamma+\cos \alpha \cos \gamma & \sin \alpha \sin \beta \cos \gamma-\cos \alpha \sin \gamma \\ -\sin \beta & \cos \beta \sin \gamma\end{array}\right)$

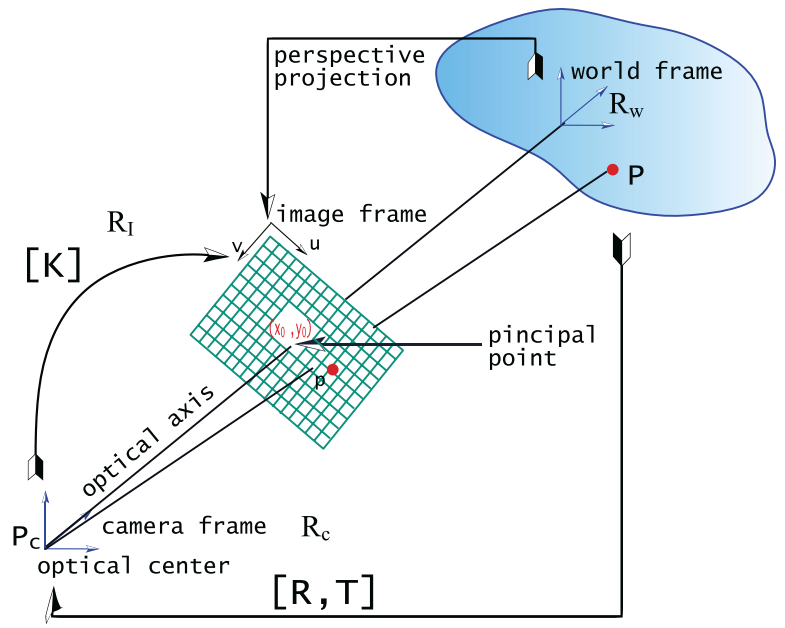

Fig. 4 Representation of the intrinsic and extrinsic parameters.

\subsection{Multiscale model}

Multiscale calibration is required because the videomicroscope works at multiple zoom or magnification. Thus it is necessary to introduce the zoom factor $\zeta$ in the above intrinsic model as followed:

$K(\zeta)=\left(\begin{array}{ccc}f(\zeta) k & 0 & x_{o} \\ 0 & f(\zeta) k & y_{o} \\ 0 & 0 & 1\end{array}\right)$

A non linear relation links the scale factor $k$ with the zoom factor $\zeta$ of the system. That relation can be represented by a polynomial relation of the from:
$k=C \cdot\left[\zeta^{10} \zeta^{2} \cdots 1\right]^{T}$

where $C=\left[c_{1} c_{2} \cdots c_{11}\right]$ is a $1 \times 11$ vector whose elements are the polynomial coefficients.

Knowing the zoom factor $\zeta$, it is possible to compute the focal length:

$f=\frac{K_{11}(\zeta)}{k}$

\subsection{Computing the parameters at multiple scale}

There are similarities between vision sensing in the macro, meso and micro scales. In each scale a $2 \mathrm{D}$ image is formed from a 3D object through an optical lens system. As they are modelled by the same model, the same approaches of calibration can be achieved. These techniques are roughly classified into two families: photogrammetric calibration approaches and self calibration approaches. In any case an important number of correspondences $p-P$ are required and the calibration is performed in two stages.

- The first stage consists in roughly estimating the projection matrix $Q$ by resolving the DLT (Direct Linear Transformation) obtained by combining the projection equations for points of correspondences.

- The second stage enables the fine estimation of the parameters by minimizing a criterion, usually the geometric error

$$
\sum_{i} d\left(p_{i}, Q P_{i}\right)
$$

Even if the same algorithm can be implemented both in macro and micro scales, there are some fundamental differences between standard lens and a microscope based image sources calibration. A microscope is characterized by weak depth of field $D O F$ and field of view FOV. As a consequence of these limitations a 3D calibration sample cannot be used, the pattern is reduced to a planar object that should be almost parallel to the lens. A videomicroscope is heavy and cumbersome and thus is not easy to manipulate. These constraints complicate the calibration of the videomicroscope. On the other hand, the presence of an accurate motion source like $x y z$ stage enables accurate motions of the plane calibration sample and contributes to facilate the calibration.

Instead of using a real pattern or virtual points, the proposition is to use the power of image processing: the center of gravity of a micrometric part is tracked in the images of the scene. The micropart is moved accurately by a $x y z$ stage, so its central position in the scene is known with high accuracy. Those positions are used to synthesize a virtual image which is used in the algorithm exposed above. This approach 
enables high accuracy in the computing of the model parameters. The approach is also simple, as it does not require the micromachining of any pattern. The components necessary for the assembling are directly used ([1]).

\section{Vision algorithms}

\subsection{Tracking of a microcomponent and an end-effector}

Image processing and vision are very important in visual servoing. The following conditions are essential for the success of the control.

- Vision should be able to deliver visual information at a rate close to the sampling frequency of the robot control,

- Interaction matrix should be explicitly calculated,

- Command must be robust to little occlusions (like the passage of the micropart under the gripper).

There are several methods of visual tracking which are usually classified into two groups. The first group consists in tracking local features like lines, segments, points, edges... ([45]). The results of these techniques depend highly on the quality of the images and remain very sensitive to feature detection ([26]). The other group contains methods that perform a comparison between two frames in image sequences by minimizing an error based on the image brightness. These methods take into account some parameters like motion, deformation or illumination parameters between the two frames or the frame and a template.

Images of the planar silicon microparts as well as those of the gripper tips are not very sharp because of the properties of the microscope. Consequently a robust tracking algorithm capable of working in real-time is required. The tracking is achieved by an algorithm proposed by Malis et al. ([26], [37]). This algorithm is based on the second-orderminimization (ESM); it has a far higher convergence rate than other techniques which is an important property for real-time tracking. For the micropart, the coordinates of the four points delimiting the bounding box of the micropart is estimated. For the above finger (figure2) the coordinates of the tip (point $\left.p_{1}=\left(u_{1}, v_{1}\right)\right)$ is estimated. That point is the bottom left point of the searching template $W_{1}(n \times m)$.

\subsection{Autofocus achievement and depth recovery}

Because of the weakness of the depth of field as exposed above, an autofocus method is implemented in order to guarantee the obtaining of focused images during the experiments. The depth of the scene is scanned step by step, images are acquired and the focus is estimated. The system is repositioned in the position where the focus estimation is highest. Three focus estimators have been tested: the variance $F_{V a r}$, the intercorrelation $F_{A}$ and the Brenner gradient $F_{B}$. The formulas are respectively:

$F_{\mathrm{Var}}=\frac{1}{H \cdot W \cdot \mu} \sum_{H} \sum_{W}(i(x, y)-\mu)^{2}$

$F_{A}=\sum_{H} \sum_{W} i(x, y) i(x+1, y)-\sum_{H} \sum_{W} i(x, y) i(x+2, y)$

$F_{B}=\sum_{H} \sum_{W}(i(x+2, y)-i(x, y))^{2}$

where $H$ and $W$ are respectively the image height and width and $\mu$ is the mean of image intensity.

The variance focus estimator is selected because it makes a trade off between resolution and the speed of calculation. The representation of the focus estimation according to $z$ motion gives two peaks (Fig. 5): the low peak corresponds to the gripper and the high peak corresponds to the platform with the component (see Fig. 6). Therefore, it is possible to determine the depth $Z_{f}$ between the camera and the micropart:

$Z_{f}=Z_{o}+Z_{g}$

where $Z_{o}$ and $Z_{g}$ are respectively the position of the micro object and the gripper along the microscope axis. This depth $Z_{f}$ is used to servo the motion of the videomicroscope on the motion of the gripper.

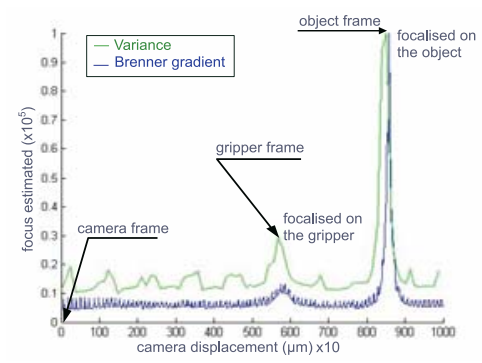

Fig. 5 Focus estimation result using the variance and Brenner Gradient methods.

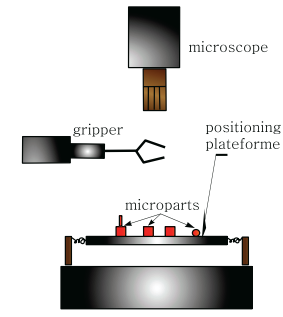

Fig. 6 schematization of the positions of the different elements: microscope, gripper and positioning platform.

\section{Experimental setup}

The above concepts were validated by performing the manipulation of silicon microparts $(400 \mu \mathrm{m} \times 400 \mu \mathrm{m} \times 100 \mu \mathrm{m})$ by means of a microassembly workcell including a robotic 
system (made by commercial positioning stages), a homemade gripping system (a two-fingers gripper) and a commercial photon videomicroscope (Leica MZ 16 A). All these elements were placed inside a controlled environment to enable the reduction of the rate of dust particles.

\subsection{Robotic system}

From a kinematic point of view, the workstation is a five dof robotic system (Fig. 7). Three dof in translation are achieved by three high accuracy linear stages and two dof in rotation are achieved by two high accuracy angular stages (all from Polytec PI). The translation motions $x, y$, and $z$ are characterized by a resolution $r=0.007 \mu \mathrm{m}$, an increment $i=0.05$ $\mu \mathrm{m}$, a velocity $v_{t}=1.5 \mathrm{~mm} / \mathrm{s}$, a stroke of $s_{t}=25 \mathrm{~mm}$. The specifications of the angular motions are: $r=26 \mu \mathrm{rad}, i=26$ $\mu \mathrm{rad}$ and speed $v_{r}=45 \mathrm{deg} / \mathrm{s}$. All the dof were distributed into two robotic systems: a $x y \theta$ system and a $z \varphi$ system. The former system (the positioning platform) was equipped with a compliant table (the table is supported by three springs) and enables the positioning in the horizontal plane. The latter system (the micromanipulator) supports the gripper and enables the vertical positioning and spatial orientation of microparts.

\subsection{Gripping system}

MMOC (Microprehensile Microrobot On Ship) gripper developed in the laboratory is used for the handling. It has four dof and allows open-and-close motions as well as up-anddown motions. Modularity is an important design criterion during development, and the MMOC microgripper was designed to use different end-effectors (finger tips) on nickel or silicon ([20]). This microgripper can grab different objects (planar silicon parts, balls, gears, optical fibers, ....). The nickel end-effector characteristics and performances are: an open-close of $320 \mu \mathrm{m}$, an up-and-down of $200 \mu \mathrm{m}$, a blocking force of $55 \mathrm{mN}$ and a resolution of $\sim 10 \mathrm{~nm}$.

\subsection{Imaging system}

The imaging system is a video stereo microscope of the type LEICA MZ 16 A vertically positioned to the work scene. It delivers a top view of the work scene. The zoom (and thus the magnification) and the focus are motorized and controlled by a PC. The field of view varies from $700 \mu \mathrm{m} \times 900 \mu \mathrm{m}$ with a resolution of $1.4 \mu \mathrm{m}$ at the maximum of the magnification to $20 \mathrm{~mm} \times 25 \mathrm{~mm}$ with a resolution of $21 \mu \mathrm{m}$ at the minimum of magnification. The depth of field varies from $2.9 \mathrm{~mm}$ to $0.035 \mathrm{~mm}$ according to the numerical aperture of the objective. The work distance is approximately $112 \mathrm{~mm}$.
The workcell is also equipped with a videomicroscope based on a long tube for the side view but the latter is not considered in this paper.

\section{Experimental results}

\subsection{Calibration results}

The optical microscope calibration involves two stages: the establishment of the relation between the scale $(k)$ and zoom $(\zeta)$ factors and the computing of the intrinsic parameters $(K)$ at a given zoom factor $(\zeta)$. A metal object with three holes is used in this stage. The diameters of the holes are estimated to $d_{1}=1961.5 \mu \mathrm{m}, d_{2}=1037.5 \mu \mathrm{m}$ and $d_{3}=429.85 \mu \mathrm{m}$ using a SEM of $r=10 \mathrm{~nm}$ resolution. According to the zoom factor the relevant hole image is considered. The zoom factor $\zeta$ (called magnification according to the supplier) is modified with a step of 0.5 and then 172 images are acquired. In every image the relevant hole is detected using the normalized correlation and the scale factor $k$ corresponding to the ratio of the diameter $\left(d_{1}, d_{2}\right.$ or $\left.d_{3}\right)$ of the hole in $\mu \mathrm{m}$ and in pixel is computed. The representation of the scale factor $(k)$ versus the zoom factor $(\zeta)$ is given in Fig. 8 .

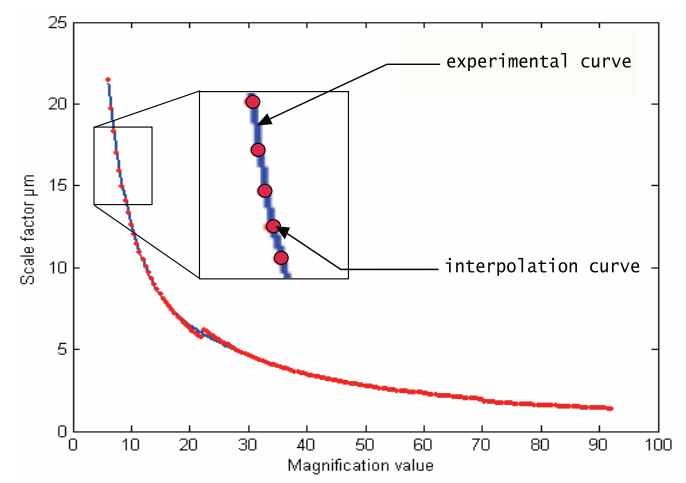

Fig. 8 Representation of the scale factor according to the magnification.

For the second stage of calibration, a planar virtual pattern is achieved from the tracking of a silicon micropart of $400 \mu \mathrm{m} \times 400 \mu \mathrm{m} \times 100 \mu \mathrm{m}$. The intrinsic and extrinsic parameters for a zoom factor of $38 \times$ are summarized in the table 1.

\subsection{Visual control results}

As exposed above the basic tasks of aligning (task 5) and centering (task 6) are performed by visual servoing with an exponential decrease of error and an adaptive gain, when the task of closing (task 9) is performed by visual control with a polynomial decrease of error. The figure 9 shows the result 


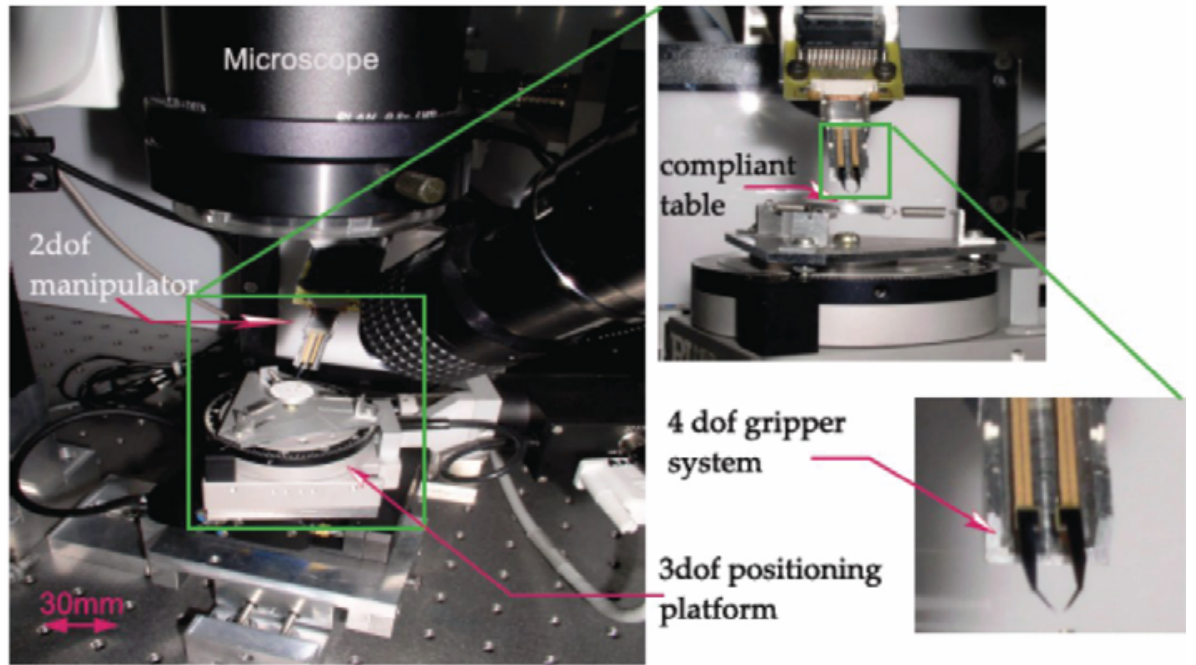

Fig. $75+4$ dof assembly workcell.

\begin{tabular}{cc}
\hline intrinsic parameters & extrinsic parameters \\
\hline$k_{x}=3.6444 \mu \mathrm{m}$ & $\alpha=88.73^{\circ}$ \\
\hline$k_{y}=3.6444 \mu \mathrm{m}$ & $\beta=-27.26^{\circ}$ \\
\hline$f=11.466 \times 10^{3} \mu \mathrm{m}$ & $\gamma=4.01^{\circ}$ \\
\hline$\left(x_{o}, y_{o}\right)=(473,337)(\mathrm{pixel})$ & $\mathbf{T}_{\mathbf{x}}=882(\mathrm{~mm})$ \\
\hline$d=13144.53(\mathrm{~mm})$ & $\mathbf{T}_{\mathbf{y}}=963(\mathrm{~mm})$ \\
\hline$M=3.8 \times$ & $\mathbf{T}_{\mathbf{z}}=13156(\mathrm{~mm})$ \\
\hline
\end{tabular}

Table 1 Summarization of the calibration results.

of the alignment of the micropart parallel to the $x$ axis (basic task 5). It can be seen that the angle decreases exponentially to zero: the final error is about 0.5 degrees. This very small value shows the relevance of the control law. It is possible to orient effectively any planar component according to the axis.

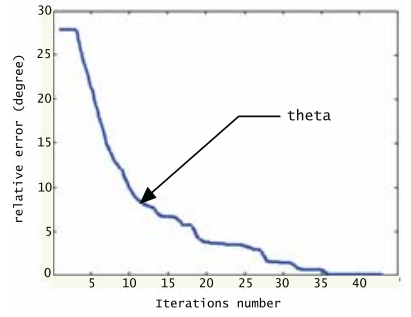

Fig. 9 Aligning error versus the number of iterations.

Figure 10 shows the result of the centering of the micropart with respect to the gripper (basic task 6). It can be seen that the implementation of an adaptive gain prevents the overshoot of the reference position. The final error is about 0.2 pixel in $x$ and $y$ directions (Fig. 11).

Figure 13 shows the micropart in the gripper after the sequence aligning-centering-descent-closing-gripping-unsticking-of the micropart with respect to the horizontal plane. the gripping task.

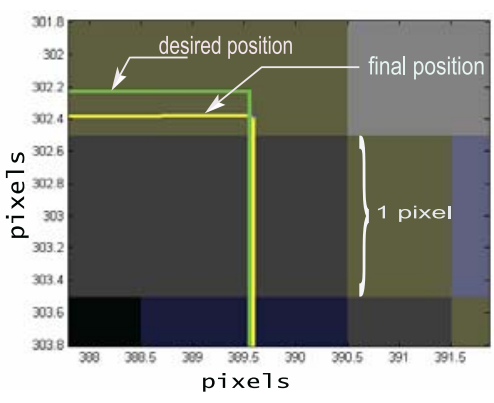

Fig. 11 Final error of centering. In yellow color, is represented the final position of the micropart and in green color the desired position.

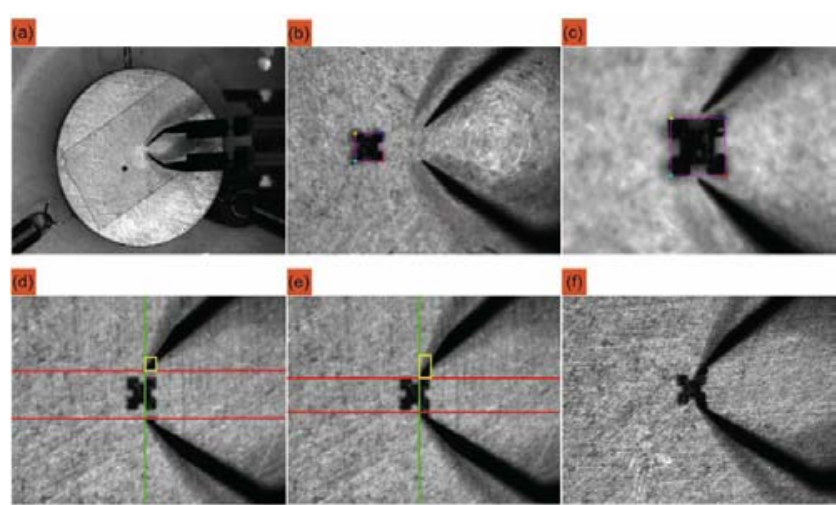

Fig. 12 Images (a), (b) and (c) show the performing of the positioning task with the increasing zoom task and images (d), (e) and (f) illustrate

ascent. This view is acquired with a second imaging system (the lateral view is not used to perform the visual control laws implemented). It can be noticed the correct orientation 


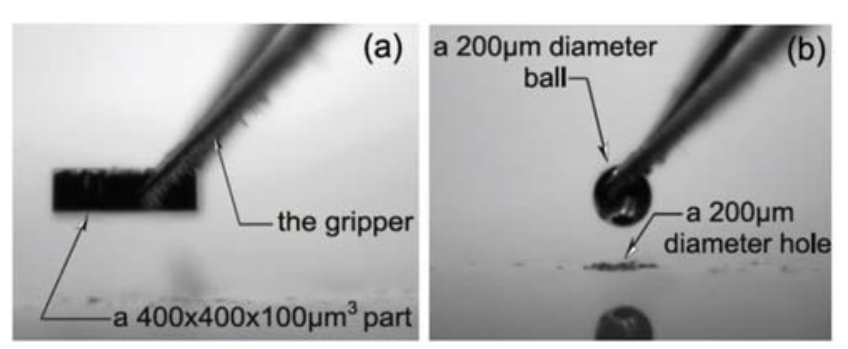

Fig. 13 Side view of two examples of gripping microparts at the task 12. Image (a) represents the gripping of a silicon micropart and image (b) show the gripping of a microball of $200 \mu \mathrm{m}$ of diameter in the gripper fingers.

\subsection{Cyclic pick-and-place results}

The sequence of tasks represented in Fig. 2 which models the pick-and-place of a silicon micropart is performed several times in order to estimate the relevance of concepts. Initial and final positions and orientations of the micropart change from cycle to cycle in order to take into account the maximum of possible cases.

Let the success rate be:

Sucess Rate $=\frac{\text { Succeed Cycles }}{\text { Total Cycles }}$

The success rate obtained is about $72 \%$. Table 2 summarizes the main cases of success and failure encountered. From this table, several conclusions can be made from these experiments, the first is the robustness of the visual tracking used. Despite the weak quality of scene images and a great occlusion, the algorithm can track the micropart. It continues to work for an occlusion of $1 / 4$ of the micropart by the gripper or outside the field-of-view of the imaging system (case 3 of table 2). Occlusion is responsible of about $17 \%$ (case 5 of table 2). The second lesson that can be retrieved is the accuracy of visual control: the mean and standard deviation of errors are summarized in table 3 . There is very fewer overshots and they don't lead to failures. The error of control is the reason for about $22 \%$ of failure (case 4 of table 2). The third conclusion to be drawn from the above results is the importance of physical phenomena which are electrostatic and capillary forces, despite the control of manipulation environment. The presence of electric charges on both microparts and fingers causes the former to move to the latter during the gripper closing and leads to the failure of the cycle. That force is responsible for about $37 \%$ (case 6 of table 2) of failure. Hydrometry is high enough in the scene to prevent the unstacking of the micropart on the gripper fingers because of capillary force. That phenomenon is responsible for about $24 \%$ (idem electrostatic) (case 7 of table 2) of failure like electrostatic force.

\begin{tabular}{|c|c|c|c|c|c|}
\hline N. & Subtask & Init. Pos. & Prec. & Note & Result \\
\hline \multirow[t]{6}{*}{1} & Alig. $(\theta)$ & $14^{\circ}$ & $0.09^{\circ}$ & & Success \\
\hline & Cent. $(x, y)$ & $1029 \mu \mathrm{m}$ & $0.43 \mu \mathrm{m}$ & & Success \\
\hline & Clos. $\left(e_{1}\right)$ & $163 \mu \mathrm{m}$ & $1.72 \mu \mathrm{m}$ & & Success \\
\hline & Asce. & $\grave{\nearrow}$ & $\dot{\nearrow}$ & & Success \\
\hline & Tran. & $\nearrow$ & $\nearrow$ & & Success \\
\hline & Rele. & $\nearrow$ & $\nearrow$ & & Success \\
\hline \multirow[t]{6}{*}{2} & Alig. $(\theta)$ & $32^{\circ}$ & $0.61^{\circ}$ & & Success \\
\hline & Cente. $(x, y)$ & $1015 \mu \mathrm{m}$ & $4.91 \mu \mathrm{m}$ & & Success \\
\hline & Clos. $\left(e_{1}\right)$ & $163 \mu \mathrm{m}$ & $1.77 \mu \mathrm{m}$ & Over. & Success \\
\hline & Asce. & $\nearrow$ & & & Success \\
\hline & Tran. & $\nearrow$ & $\nearrow$ & & Success \\
\hline & Rele. & $\pi$ & $\nearrow$ & & Success \\
\hline \multirow[t]{6}{*}{3} & Alig. $(\theta)$ & $21^{\circ}$ & $0.27^{\circ}$ & & Success \\
\hline & Cente $(x, y)$ & $1321.5 \mu \mathrm{m}$ & $0.77 \mu \mathrm{m}$ & Occu. & Success \\
\hline & Clos. $\left(e_{1}\right)$ & $163 \mu \mathrm{m}$ & $1.44 \mu \mathrm{m}$ & & Success \\
\hline & Asce. & $\nearrow$ & $\check{\nearrow}$ & & Success \\
\hline & Tran. & $\nearrow$ & $\nearrow$ & & Success \\
\hline & Rele. & $\nearrow$ & $\nearrow$ & & Success \\
\hline \multirow[t]{6}{*}{4} & Alig. $(\theta)$ & $7.53^{\circ}$ & $0.68^{\circ}$ & & Success \\
\hline & Cent. $(x, y)$ & $992.5 \mu \mathrm{m}$ & $6.16 \mu \mathrm{m}$ & & Failure \\
\hline & Clos. $\left(e_{1}\right)$ & $x$ & $\times$ & & Failure \\
\hline & Asce. & $x$ & $x$ & & Failure \\
\hline & Tran. & $\times$ & $\times$ & & Failure \\
\hline & Rele. & $x$ & $x$ & & Failure \\
\hline \multirow[t]{6}{*}{5} & Alig. $(\theta)$ & $33.8^{\circ}$ & $x$ & Occu. & Failure \\
\hline & Cent. $(x, y)$ & 1.75 & $x$ & & Failure \\
\hline & Clos. $\left(e_{1}\right)$ & $163 \mu \mathrm{m}$ & $x$ & & Failure \\
\hline & Asce. & $x$ & $x$ & & Failure \\
\hline & Tran. & $x$ & $x$ & & Failure \\
\hline & Rele. & $x$ & $x$ & & Failure \\
\hline \multirow[t]{6}{*}{6} & Alig. $(\theta)$ & $13^{\circ}$ & $0.24^{\circ}$ & & Success \\
\hline & Cente. $(x, y)$ & $560.7 \mu \mathrm{m}$ & $0.86 \mu \mathrm{m}$ & & Success \\
\hline & Clos. $\left(e_{1}\right)$ & $163 \mu \mathrm{m}$ & $x$ & $\mathrm{EF}$ & Failure \\
\hline & Asce. & $x$ & $x$ & & Failure \\
\hline & Tran. & $x$ & $\times$ & & Failure \\
\hline & Rele. & $x$ & $x$ & & Failure \\
\hline \multirow[t]{6}{*}{7} & Alig. $(\theta)$ & $15.5^{\circ}$ & $0.52^{\circ}$ & & Success \\
\hline & Cent. $(x, y)$ & $348.2 \mu \mathrm{m}$ & $0.64 \mu \mathrm{m}$ & & Success \\
\hline & Clos. $\left(e_{1}\right)$ & $163 \mu \mathrm{m}$ & $1.11 \mu \mathrm{m}$ & & Success \\
\hline & Asce. & $\dot{\nearrow}$ & $\dot{\nearrow}$ & & Success \\
\hline & Tran. & $\nearrow$ & $\nearrow$ & & Success \\
\hline & Rele. & $x$ & $x$ & $\mathrm{CF}$ & Failure \\
\hline
\end{tabular}

Table 2 Results of experiments: [ $\nearrow$ ] represents the success of the task, $[\times]$ represents the failure of the task, [Occu.] represents the case when the micropart passed under the gripper, [Over.] indicates the presence of an overshot in the control, [EF] indicates the presence of important electrostatic effects between the gripper and the micropart and [CF] indicates the presence of an important capillary forces preventing release of the micropart.

\begin{tabular}{ccc}
\hline Task & Mean error & Standard deviation \\
\hline Aligning $(\theta)$ & $0.37^{\circ}$ & $0.31^{\circ}$ \\
\hline Centering $(x, y)$ & $1.87 \mu \mathrm{m}$ & $1.61 \mu \mathrm{m}$ \\
\hline Closing $\left(e_{1}\right)$ & $1.36 \mu \mathrm{m}$ & $0.34 \mu \mathrm{m}$ \\
\hline
\end{tabular}

Table 3 Linear and angular motions characteristics.

\section{Conclusion}

The robotic assembly of micrometric parts is usually investigated from an experimental point of view by reporting ex- 
periments of manipulation on microparts or on assembly. This paper addresses micromanipulation from a conceptual point of view which leads to practical solutions of achievement. The problem is conceptualized and validated by actual experiments.

Manipulation of microparts includes their handling and positioning by means of a robotic system, gripping system and imaging system. The analysis of that complex task leads to its decomposition into a set of basic tasks relative to the above systems. Fourteen tasks can be identified from autofocusing achievement, increasing the imaging scale to gripping, transfer and release of the micropart. Several logical combinations of these basic tasks lead to the achievement of complex task. Each sequence can be usefully represented by a sequential function chart. A pre-defined sequence for a task of pick-and-place is proposed to illustrate our research but for more advanced tasks it would be interesting to determine the best sequence as in the case of assembly on a conventional scale.

It is proposed to use this manipulation sequence as a model for the behavior of systems achieving the task. As a consequence that achievement becomes a sequence of servoings especially visual controls. As the photon imaging system works at multiple scale in order to resolve the fieldof-view/resolution dilemma, its multiple scale modelling is proposed. It consists in explicitly establishing the relation linking scale factor $k$ with the zoom (or magnification) factor $(\zeta)$ and computing the projective model $(\mathrm{K})$ for a given zoom factor $\left(\zeta^{*}\right)$. The corresponding calibration approach combines robotics and vision: an interesting point of a part whose motion is known is tracked in the images of the scene and combined to define a virtual calibration pattern. Consequently, the visual servoing law of the type exponential decrease of error is modified to integrate the scale change through the zoom factor.

Pick-and-place of silicon micropart of $400 \mu \mathrm{m} \times 400$ $\mu \mathrm{m} \times 100 \mu \mathrm{m}$ and the following systems are used to test the concepts: a robotic system including a $x y \theta$ and $\varphi \mathrm{z}$ subsystems, a two-finger gripper, a photon videomicroscope with controllable zoom (magnification from $0.7 \times$ to $11.5 \times$ ) and focus. That videomicroscope is calibrated and the results are used to implement the basic task of aligning, centering and gripper closing by visual control. The results of experiments demonstrate the relevance of the concepts. Indeed, the failure rate of $30 \%$ comes mainly from the physical phenomena and few from control error or occlusions. Despite the controlled environment there are a lot of electrical charges which cause the part to be attracted by the gripper finger and a lot of humidity which prevents the release of the micropart by the gripper.

The next stage of the work will concern the improving of the efficiency of the controlled environment in order to drastically reduce the effects of physical phenomena and the implementation of trajectory planning to avoid occlusions of the micropart by the gripper and its exit from the field-ofview. The concepts will be extended to the task of insertion enabling the achievement of $3 \mathrm{D}$ compound products.

\section{AKNOWLEDGEMENTS}

This work is partially conducted with financial support from the project Hybrid Ultra Precision Manufacturing Process Based on Positional and Self assembly for Complex MicroProducts (HYDROMEL NMP2-CT-2006-026622) funded by the European Commission.

\section{References}

1. M. Ammi, V. Fremont, and A. Ferreira, Flexible microscope calibration using virtual pattern for 3-d telemicromanipulation, IEEE ICRA, (Spain), pp. 3888-3893, (apr., 2005).

2. K. Aoki, H. T. Miyazaki, H. Hirayama, K. Inoshita, T. Baba, K. Sakoda, N. Shinya, and Y. Aoyagi, Microassembly of semiconductor three-dimensional photonic crystals, Nature Materials vol. 2 (2), pp. 117-121, (2003).

3. K. Ashida, N. Mishima, H. Maekawa, T. Tanikawa, K. Kaneko, and M. Tanaka, Development of desktop machining microfactory, Proc. of the Japan-USA Symp. on Flexible Assembly, (2000).

4. F. Chaumette and S. Hutchinson, Visual servo control, part 2: Advanced approaches, IEEE Rob. and Auto. Magazine, vol. 14, no. 1, pp. 109-118, (2007).

5. F. Chaumette and S. Hutchinson, Visual servo control, part 1: Basic approaches, IEEE Rob. and Auto. Magazine vol. 13, no. 4, pp. 82-90, (2006).

6. L. Chen, W. R. Sun, L. and, and X. Bian, Hybrid control of vision and force for mems assembly system, IEEE Int. Conf. on Rob. and Biometrics, (China), pp. 136-141, (2004).

7. S. Koelemeijer Cholet, F. Bourgeois, L. Benmayor, B. Moll, C. Wulliens, and J. Jacot, A flexible microassembly cell for small and medium sized batches, Proc.of the 33 rd Int. Symp. on Rob., (2002).

8. N. Dechev, W. L. Cleghorn, and J. K. Mills, Microassembly of 3$d$ microstructures using a compliant, passive microgripper, Jour. of Microelectromechanical Systems vol. 13, no 2, pp.176-189, (2004).

9. N. Dechev, W. L. Cleghorn, and J. K. Mills, Construction of $3 d$ mems microstructures using robotic microassembly, Int. Conf. of Intel. Rob. and Sys., (2003).

10. R. Devanathan, S. Wenting, S. Chai, and A. Shacklock, Multi view and multi scale image based visual servo for micromanipulation, Studies in Comput. Intel.vol. 8, pp. 105-133, (2006).

11. E. T. Enikov, S. Clark, and L. Minkov, Micro-assembly cell with dual optical/computer vision control for electrostatic gripping of mems, SPIE vol. 5267, pp. 269-280, (2003).

12. E. T. Enikov and L. Minkov, Micro-assembly and packaging of mems using optically transparent electrostatic gripper, Proc. of Int. Mech. Eng. Congr. and Exposition, (USA), (2004).

13. S. Fatikow, J. Seyfried, ST. Fahlbuschand A. Buerkle, and F. Schmoeckel, A flexible microrobot-based microassembly station, Jour. of Intel. and Rob. Sys., vol. 27, pp. 135-169, (2000).

14. R. S. Fearing, Survey of sticking effects for micro parts handling, IEEE IROS, (Pittsburgh, PA), pp. 212-217, (1995).

15. J. T. Feddema and R. W. Simon, Visual servoing and cad-driven microassembly, IEEE Rob. and Auto. Magazine vol. 5 (4), pp. 1824, (1998). 
16. M. Figl, C. Ede, J. Hummel, F. Wanschitz, R. Ewers, H. Bergmann, and W. Birkfellner, A fully automated calibration method for an optical see-through head-mounted operating microscope with variable zoom and focus, IEEE Trans. on Med. Imaging vol. 24, no. 11, pp. 1492-1499, (2005).

17. T. Fukuda and F. Arai, Micromanipulation and robotic technology, Int. Conf. on modeling and simulations of microsystems, (1998).

18. J. A. Gaines Ge Yang and B. J. Nelson, A surpervisory wafer-level $3 d$ microassembly system for hybrid mems fabrication, Jour. of Intel. and Robo. Sys., vol. 37, pp. 43-68,(2003).

19. M. Ghovanloo and K. Najafi, A three-dimensional microassembly structure for micromachined planar microelectrode arrays, Proc. of the 3rd Annual Int. IEEE EMBS Special topic Conf. on Microtechnologies in medecine and biology, (Hawaii), pp. 112- 115, (2005).

20. J. Agnus, P. Nectoux, and N. Chaillet, Overview of microgrippers and design of a micromanipulation station based on mmoc microgripper, IEEE Int. Symp. on Computational Intel. in Rob. and Auto., (Finland), pp. 117-123, (2005).

21. B. Kim, H. Kang, D. -Ho Kim, and J. -Oh Park, A flexible microassembly system based on hybrid manipulations scheme for manufacturing photonics components, Int. Jour. of Advanced Manufacturing Technology, vol. 28, pp. 379-386, (2006).

22. K. Kim, D. -Ho Kim, S. J.. Lee, and J. Lee, Hybrid microassembly system for three-dimensional mems components, IWMF, (Minneapolis, Minnesota), (2002).

23. S. J. Lee, K. Kim, D. -Ho Kim, J. -Oh Park, and G. -T. Park, Vision based micromanipulation, IWMF, (Minneapolis, Minnesota), (2002).

24. S. -J. Lee, K Kim, D -Ho Kim, J -Oh Park, and G -Tae Park, Multiple magnification images based micropositioning for $3 d$ micro assembly, ICARCV, pp. 914-919 (2002).

25. X. Lv and X. Huang, Three-layered control architecture for microassembly with human-robot task plan interaction, IEEE Int. Conf. on Rob. and Biometrics, (China), pp. 617-622, (2004).

26. E. Malis, Improving vision-based control using efficient secondorder minimization techniques, IEEE Int. Conf. on Rob. and Auto., (New-Orleans, USA), pp. 1843-1848, (2004).

27. B. J. Nelson, S. Ralis, Yu Zhou, and B. Vikramaditya,Force and vision feedback for robotic manipulation of the microworld, Experimental Rob. - VI-Lectures Notes in Control and Information Sciences vol. 250, pp. 433-442, (1999).

28. H. Ogawa, Indispensable technologies for microassembly, Proc. of the 2nd Int. Workshop on MicroFactories, (2000).

29. Y. Okazaki, N. Mishima, and K. Ashida, Microfactory - concept, history and developments, Jour. of Manufacturing Science and Eng., vol. 126, pp. 837-844, (2004).

30. D. O. Popa, B. H. Kang, J. Sin, and J. Zou, Reconfigurable microassembly system for photonics applications, IEEE Int. Conf. on Rob. and Auto., (USA), pp. 1495-1500, (2002).

31. D. O. Popa and H. E. Stephanou, Micro and mesoscale robotic assembly, Jour. of Manufacturing Process, vol. 6 (1), pp. 52-71, (2004).

32. M. Probst, R. Borer, and B. J. Nelson, A microassembly system for manufacturing hybrid mems, IFTOMM World Congress, (France), (2007).

33. S. Ralis, B. Vikramaditya, and B. J. Nelson, Micropositioning of a weakly calibrated microassembly system using coarse-to-fine visual servoing strategies, IEEE Trans. on Elec. Packaging Manufacturing, vol. 23 (2), pp. 123-131, (2000).

34. R. De La Rue, Photonic crystals: Microassembly in 3d, Nature Materials, vol. 2 (2), pp. 74-76, (2003).

35. R. Saini, Z. Jandric, K. Tsui, T. Udeshi, and D. Tuggle, Manufacturable mems microcolumn, Jour. of Microelectronic Eng., vol. 7879, pp. 62-72, (2005).

36. A. Sieber, P. Valdastri, K. Houston, A. Menciassi, and . Dario, Flip chip microassembly of a silicon triaxial force sensor on flexible substrates, Sensors and Actuators A vol. 142, pp. 421-428, (2008).
37. G. Silveira and E. Malis, Real time tracking under arbitrary illumination changes, In Comp. Vis. and Pat. Reco., pp. 1-6, (2007).

38. L. Sun, H. Xie, W. Rong, and L. Chen, Task-reconfigurable system for mems assembly, ICRA, (Spain), (2005).

39. W. T. Sun and T. C. Chin, Image-based visual servo for micromanipulation: a multiple-view and multiple-scale approach, Int. Sym. on Micro-Nanomechatronics and Human Science, pp. 341346, (2004).

40. Y. Sun, S. Duthaler, and B. J. Nelson, Autofocusing algorithm selection in computer microscopy, IROS, pp. 70-76, (2005).

41. X. Tao, H. Cho, and Y. Cho, Microassembly of peg and hole using active zooming, SPIE vol. 6052, pp. 1-12, (2005).

42. K. Tsuchiya, A. Murakami, G. Fortmann, M. Nakao, and Y. Hatamura, Micro assembly and micro bonding in nano manufacturing world, SPIE, vol. 3834, pp. 132-140, (1999).

43. T. Udeshi and K. Tsui, Assembly sequence planning for automated micro assembly, ISATP, pp. 98-105, (2005).

44. B. Vikramaditya and B. J. Nelson, Visually servoed micropositioning for robotic micromanipulation, Microcomputer Applications, vol. 18, pp. 23-31, (1999).

45. X. Liu W. Wong and Y. Sun, Robust contact detection in micormanipulation using computer vision microscopy, IEEE Eng. Med. Biol. Soc. pp. 2219-2222, (2006).

46. J. Wason, W. Gressick, J. T. Wen, J. Gorman, and N. Dagalakis, Multi-probe microassembly, IEEE Conf. on Auto. Science and Eng., (USA), pp. 22-25, (2007).

47. H. Xie, W. Rong, and L. Sun, A flexible experimental system for complex microassembly under microscale force and vision-based control, Int. Jour. of Optomechatronics, vol. 1 (1), pp. 81-102, (2007).

48. G. Yang, J. A. Gaines, and B. J. Nelson, Optomechatronic design of microassembly systems for manufacturing hybrid microsystems, IEEE Trans. on Industrial Elect., vol. 52 (4), pp. 1013-1023, (2005).

49. Y. Zhou and B. J. Nelson, Calibration of a parametric model of an optical microscope, Optical Eng., vol. 38, no. 12, pp. 1989-1995, (1999).

50. Y. Zhou, B. J. Nelson, and B. Vikramaditya, Integrating optical force sensing with visual servoing for microassembly, Jour. of Intel. and Rob. Sys., vol. 28, pp. 259-276, (2000). 